\title{
Wind-wave amplification mechanisms: possible models for steep wave events in finite depth
}

\author{
P. Montalvo ${ }^{1,2}$, R. Kraenkel ${ }^{3}$, M. A. Manna ${ }^{1,2}$, and C. Kharif ${ }^{4,5}$ \\ ${ }^{1}$ Université Montpellier 2, Laboratoire Charles Coulomb, UMR5221, 34095, Montpellier, France \\ ${ }^{2}$ CNRS, Laboratoire Charles Coulomb, UMR5221, 34095, Montpellier, France \\ ${ }^{3}$ Instituto de Física Téorica, UNESP - Universidade Estadual Paulista, Rua Dr. Bento Teobaldo Ferraz 27, Bloco II, \\ 01140-070, São Paulo, Brazil \\ ${ }^{4}$ Ecole Centrale Marseille, 38 rue Frédéric Joliot-Curie, 13451 Marseille cedex 20, France \\ ${ }^{5}$ Institut de Recherche sur les Phénomènes Hors Équilibre, CNRS, AMU, UMR7342, 49 rue Frédéric Joliot-Curie, BP 146, \\ 13384 Marseille cedex 13, France
}

Correspondence to: M. A. Manna (miguel.manna@univ-montp2.fr)

Received: 10 June 2013 - Published in Nat. Hazards Earth Syst. Sci. Discuss.: 8 July 2013

Revised: 1 October 2013 - Accepted: 10 October 2013 - Published: 8 November 2013

\begin{abstract}
We extend the Miles mechanism of wind-wave generation to finite depth. A $\beta$-Miles linear growth rate depending on the depth and wind velocity is derived and allows the study of linear growth rates of surface waves from weak to moderate winds in finite depth $h$. The evolution of $\beta$ is plotted, for several values of the dispersion parameter $k h$ with $k$ the wave number. For constant depths we find that no matter what the values of wind velocities are, at small enough wave age the $\beta$-Miles linear growth rates are in the known deep-water limit. However winds of moderate intensities prevent the waves from growing beyond a critical wave age, which is also constrained by the water depth and is less than the wave age limit of deep water. Depending on wave age and wind velocity, the Jeffreys and Miles mechanisms are compared to determine which of them dominates. A wind-forced nonlinear Schrödinger equation is derived and the Akhmediev, Peregrine and Kuznetsov-Ma breather solutions for weak wind inputs in finite depth $h$ are obtained.
\end{abstract}

\section{Introduction}

The pioneer theories to describe surface wind-wave growth in deep water began with the works of Jeffreys (1925), Phillips (1957) and Miles (1957, 1997), and the modern investigations take nonlinearity and turbulence effects into account. Janssen (2004) has provided a thorough review of the topic.

\subsection{Miles' and Jeffreys' mechanisms of wind-wave growth}

Miles' and Jeffreys' theories consider both air and water to be incompressible and disregard viscosity effects. Both theories are linear and Miles' mechanism is limited to the deepwater domain. They give the linear wave growth $\gamma_{\text {Miles }}$ and $\gamma_{\text {Jeffreys }}$ (respectively noted $\gamma_{\mathrm{M}}$ and $\gamma_{\mathrm{J}}$ ) of wind-generated normal Fourier modes of wave number $k$. In Miles' theory the basic state is a shear current in air and still water. The air turbulence is disregarded (Janssen, 2004) aside from establishing the logarithmic profile of the wind flow. The Miles mechanism of wave generation by wind states that waves are produced and amplified through a resonance phenomenon. Resonance appears between the wave-induced pressure gradient on the inviscid airflow and the surface waves. The resonant mechanism happens at a critical height where the airflow speed matches the phase velocity of the surface wave.

In 1925, Jeffreys produced the first plausible mechanism to explain the necessary shift of the atmospheric pressure required for an energy transfer from the wind to the waves. He assumed that this energy transfer was solely caused by a combination of form drag and flow separation. It was assumed that flow separation occurred on the leeward side of wave crests with re-attachment further down on the leeward slopes of the wave. So flow separation is central in Jeffrey's theory. The resulting growth rate $\gamma_{\mathrm{J}}$ increases with the difference between the wave phase velocity and the wind speed.

Published by Copernicus Publications on behalf of the European Geosciences Union. 
Jeffreys computed $\gamma_{\mathrm{J}}$ for deep water (Jeffreys, 1925) and for finite depth as well (Jeffreys, 1926). Airflow separation occuring only over steep waves (Banner and Melville, 1976; Kawai, 1982), the Jeffreys sheltering mechanism must be applied locally in time and space rather than constantly and everywhere on the wave field. Note that the sheltering mechanism is working even without proper airflow separation. In fact, there is a thickening of the boundary layer (Reul et al., 2008) on the leeward side that generates a pressure asymmetry and consequently a sheltering effect.

\subsection{The Miles and Jeffreys mechanisms in finite depth: basis to model freak wave events in coastal regions}

Generally extreme wave events occur in the presence of wind. Kharif et al. (2008) and Touboul and Kharif (2006) investigated the influence of wind on extreme wave events using the Jeffreys sheltering theory. They have shown that extreme events may be sustained longer by the airflow separation. This mechanism can only be invoked if the wave is steep enough to effectively separate the airflow. Otherwise, for a too-low steepness parameter ka the Jeffreys sheltering mechanism due to flow separation becomes irrelevant.

Miles' and Jeffreys' mechanism of wind-generated surface waves in deep water was used in Touboul et al. (2008) to describe (theoretically and numerically) the evolution of a chirped wave packet under wind forcing. A comparison of the $\gamma_{\mathrm{M}}$ and the $\gamma_{\mathrm{J}}$ values corresponding to a modified Jeffreys mechanism was developed.

However, all this work is limited to deep water and hence unable to fully describe winds generating nearshore waves where the wave field is influenced by bottom bathymetry. Consequently they are not adequate to correctly describe the wind influence on extreme wave events in the coastal zone.

Therefore an extension of Miles' theory of wind-generated monochromatic waves to the case of finite depth under weak or moderate winds is needed, as well as a theoretical formulation of Jeffreys' theory in terms of adequate finite depth parameters.

\subsection{Nearshore extreme wave events}

Extreme wave events are anomalous large-amplitude surface waves. They are called freak or rogue waves. It is crucial to understand the physical mechanisms producing freak waves, as well as to obtain an accurate prediction of their dynamics in extreme sea states. A number of mechanisms generating freak waves have been identified. Such mechanisms are, for instance, linear space-time focusing, variable currents, interaction waves/currents, modulational or BenjaminFeir (BF) instability. A classical way to model the BF instability is to use the nonlinear Schrödinger equation (NLS), as in references Touboul and Kharif (2006), Touboul et al. (2008), Kharif et al. (2008) and Onorato and Proment (2012).
However, in these papers, the NLS equation was used in deep water. In a recent paper Didenkulova, Nikolkina and Pelinovsky (2013) have described and compared properties of rogue waves in intermediate depth with those in deep water. The focused regime of the BF instability was studied using a NLS equation in arbitrary depth.

In this work we are able to produce an adequate model for nearshore extreme wave events. This is done using our finite depth extensions of Miles' theory and the NLS equation in finite depth under the wind action. The case of a Jeffreys type wind input is straightforward.

NLS is exactly solvable, and some of its deterministic solutions are good candidates to be weakly nonlinear prototypes of rogue waves in finite depth under wind input. This is the case of the Akhmedian, Peregrine and Kusnetsov-Ma solutions.

The aims of this paper are: (i) to extend the Miles mechanism to a finite depth setup, (ii) to express the Jeffreys mechanism in terms of adequate dimensionless parameters, (iii) to determine, in terms of wave age and wind velocity, which among the Miles and Jeffreys mechanisms prevails and (iv) to derive a wind-forced nonlinear Schrödinger equation (NLS) in finite depth to study the effects of wind and depth on extreme wave events due to the modulational instability.

The paper is organized as follows. In Sects. 2, 2.1 and 2.2 the linear stability problem of the air-water interface is presented and the derivation of the system of equations coupling the waves to the airflow with the corresponding dispersion relation is done. In Sects. 3, 3.1 and 3.2, we write the growth rates of Miles' and Jeffreys' theories, with appropriate scalings and variables. In Sect. 4.1 the Miles coefficient $\beta$ is plotted as a function of wave age with $k h$ constant. Next in Sect. 4.2, we present our results about the evolution of the growth rate as a function of the wave age with $h$ constant for several wind velocities. A comparison between Miles' and Jeffreys' theory is shown and discussed in Sect. 4.3. In Sect. 5 we derive a wind-forced NLS equation in finite depth $h$. The Akhmediev, Peregrine and Kuznetsov-Ma breather solutions for weak wind inputs in finite depth $h$ are exhibited. Finally in Sect. 6, conclusion and perspectives are drawn.

\section{Coupling of the air and water dynamics at the interface}

The fluid particle coordinates are expressed in a fixed 3dimensional Cartesian frame $O x y z, O z$ being the upwards axis. We assume the problem to be invariant on the $y$ axis, reducing the problem to an area parallel to $O x z$. We define the surface $z=0$ as the rest state of the interface. The interface perturbation itself is denoted by $\eta$ and depends on $(x, t)$. The water depth is set at $-h$, and the air extends from the interface to infinity. 


\subsection{Water dynamics}

The horizontal and vertical components of the fluid velocity are $u$ and $w$, both depending on $(x, z, t)$. They obey the linear Euler equations of motion in finite depth (Lighthill, 1978):

$u_{x}+w_{z}=0, \quad \rho_{\mathrm{w}} u_{t}=-P_{x}, \quad \rho_{\mathrm{w}} w_{t}=-P_{z}$,

$w(-h)=0 \quad$ at $\quad z=-h$,

$\eta_{t}=w(0) \quad$ at $\quad z=\eta(x, t)$,

$P(\eta)=P_{\mathrm{a}}(\eta)+\rho_{\mathrm{w}} g \eta-P_{0} \quad$ at $\quad z=\eta(x, t)$.

Here $P=p+\rho_{\mathrm{w}} g z-P_{0}$ is a reduced pressure with $p$ the pressure, $P_{0}$ the total atmospheric pressure, $g$ the gravitational acceleration and $\rho_{\mathrm{w}}$ the water density. $p$ and $P$ depend on $(x, z, t)$ as well, and subscripts in $u, w$ and $P$ denote partial derivatives. We solve the linear equations system Eqs. (1)-(3) with

$P=\mathcal{P}(z) e^{i \theta}, \quad u=\mathcal{U}(z) e^{i \theta}$,

$w=\mathcal{W}(z) e^{i \theta}, \quad \eta=\eta_{0} e^{i \theta}$,

where $\mathcal{P}, \mathcal{U}$ and $\mathcal{W}$ are to be found. We have the phase $\theta=$ $k(x-c t)$ with $k$ the wave number and $c$ the phase speed. $\eta_{0}$ is an unknown constant. Using Eqs. (1)-(3) we obtain $u, w$ and $P$ for all $(x, z, t)$. Then, using Eq. (4) we derive

$\rho_{\mathrm{w}} \eta_{0} e^{i \theta}\left\{c^{2} k \operatorname{coth} k h-g\right\}+P_{0}=P_{\mathrm{a}}(\eta)$.

In the Archimedean case, $P_{\mathrm{a}}(\eta)=P_{0}$ and Eq. (6) returns the classical phase speed expression,

$c^{2}=c_{0}^{2}=\frac{g}{k} \tanh (k h)$.

We need an expression for $P_{\mathrm{a}}$ at $z=\eta$ to obtain the modified $c$.

\subsection{Air dynamics}

We examine the steady state of a prescribed horizontal airflow, with a mean velocity $U$ depending only on $z$. We denote physical quantities in the air domain by a subscript a. The air density is $\rho_{\mathrm{a}}$; the perturbations to this steady state are assumed to be $u_{\mathrm{a}}, w_{\mathrm{a}}$ for the velocities and $p_{\mathrm{a}}$ for the nonreduced pressure, all quantities depending on $(x, z, t)$. Now, using a reduced pressure $P_{\mathrm{a}}=p_{\mathrm{a}}+\rho_{\mathrm{a}} g z-P_{0}$, we have

$u_{\mathrm{a}, x}+w_{\mathrm{a}, z}=0$,

$\rho_{\mathrm{a}}\left[u_{\mathrm{a}, t}+U(z) u_{\mathrm{a}, x}+U^{\prime}(z) w_{\mathrm{a}}\right]=-P_{\mathrm{a}, x}$,

$\rho_{\mathrm{a}}\left[w_{\mathrm{a}, t}+U(z) w_{\mathrm{a}, x}\right]=-P_{\mathrm{a}, z}$,

with primes indicating differentiation with respect to $z$. Next we assume $P_{\mathrm{a}}=\mathcal{P}_{\mathrm{a}}(z) e^{i \theta}, u_{\mathrm{a}}=\mathcal{U}_{\mathrm{a}}(z) e^{i \theta}, w_{\mathrm{a}}=\mathcal{W}_{\mathrm{a}}(z) e^{i \theta}$ and we add the boundary conditions on $\mathcal{W}_{\mathrm{a}}$ and $\mathcal{P}_{\mathrm{a}}$,

$$
\begin{aligned}
& \lim _{z \rightarrow+\infty}\left(\mathcal{W}_{\mathrm{a}}^{\prime}+k \mathcal{W}_{\mathrm{a}}\right)=0, \\
& \lim _{z \rightarrow z_{0}} \mathcal{W}_{\mathrm{a}}=W_{0}, \\
& \lim _{z \rightarrow+\infty} \mathcal{P}_{\mathrm{a}}=0
\end{aligned}
$$

where $W_{0}$ is a wind forcing at the surface level. It ensures that there is always an interaction between the wind and the free surface. The pressure and the wind perturbations vanish at high altitudes. For the air, there is a kinematic condition as well at the aerodynamic sea surface roughness $z_{0}$, over the free surface $\eta$. In this work, $z_{0}$ will be determined by the Charnock relation (Charnock, 1955):

$z_{0}=\alpha_{\mathrm{c}} \frac{u_{*}^{2}}{g}$

where $u_{*}$ is the friction velocity, and assuming that $\alpha_{\mathrm{c}}=$ 0.018 remains constant. The kinematic boundary condition reads

$\eta_{t}+U\left(z_{0}\right) \eta_{x}=w_{\mathrm{a}}\left(z_{0}\right)$.

Our steady airflow $U(z)$ is set as the logarithmic wind profile

$U(z)=U_{1} \ln \left(z / z_{0}\right), \quad U_{1}=\frac{u_{*}}{\kappa}, \quad \kappa \approx 0.41$,

where $\kappa$ is the Von Kármán constant. Such a profile is a common ground to describe wind phenomena close to the marine boundary layer (Garratt et al., 1996). Hence, we can reduce Eq. (15) to

$\eta_{t}=w_{\mathrm{a}}\left(z_{0}\right)$.

Then, using Eqs. (8)-(10) and (13) we obtain

$$
\begin{aligned}
& w_{\mathrm{a}}=\mathcal{W}_{\mathrm{a}} e^{i \theta}, \\
& u_{\mathrm{a}}=\frac{i}{k} \mathcal{W}_{\mathrm{a}, z} e^{i \theta}, \\
& P_{\mathrm{a}}=i k \rho_{\mathrm{a}} e^{i \theta} \int_{z}^{\infty}\left[U\left(z^{\prime}\right)-c\right] \mathcal{W}_{\mathrm{a}}\left(z^{\prime}\right) \mathrm{d} z^{\prime} .
\end{aligned}
$$

The Rayleigh equation (Rayleigh, 1880) is then found by eliminating $P_{\mathrm{a}}$ from Eqs. (9)-(10), and is valid as long as $z>z_{0}$

$(U-c)\left(\mathcal{W}_{\mathrm{a}}^{\prime \prime}-k^{2} \mathcal{W}_{\mathrm{a}}\right)-U^{\prime \prime} \mathcal{W}_{\mathrm{a}}=0$.

This equation contains a singularity at a so-called critical height $z_{\mathrm{c}}$, where $U\left(z_{\mathrm{c}}\right)=c$. All turbulence phenomena being disregarded, any possible eddies are assumed to be set below $z_{0}$, and their influence is not taken into account. We note that $\mathcal{W}_{\mathrm{a}}(z)$ and $c$ are unknown in Eqs. (18)-(21). So, we have to evaluate $P_{\mathrm{a}}(\eta)$ to get $c$. We then have

$p_{\mathrm{a}}(\eta)=P_{0}-\rho_{\mathrm{a}} g \eta+i k \rho_{\mathrm{a}} e^{i \theta} \int_{z_{0}}^{\infty}[U(z)-c] \mathcal{W}_{\mathrm{a}}(z) \mathrm{d} z$,

where $z=z_{0}$ replaces $z=\eta$ as the lower integral bound, since we are studying the linear problem. Finally, eliminating the term $i k \rho_{\mathrm{a}} e^{i \theta}$ using Eq. (17), we derive

$g(1-s)+c \frac{s k^{2}}{W_{0}} I_{1}-c^{2}\left\{\frac{s k^{2}}{W_{0}} I_{2}+k \operatorname{coth}(k h)\right\}=0$, 
where $s=\rho_{\mathrm{a}} / \rho_{\mathrm{w}}$ and $I_{1}=\int_{z_{0}}^{\infty} U \mathcal{W}_{\mathrm{a}} \mathrm{d} z, I_{2}=\int_{z_{0}}^{\infty} \mathcal{W}_{\mathrm{a}} \mathrm{d} z$. As the ratio density $s$ is of order of magnitude $10^{-3}$, we can develop the wave speed in Eq. (23) as $c=c_{0}+s c_{1}+o\left(s^{2}\right)$. Next, we find $c_{1}$ as a function of $\mathcal{W}_{\mathrm{a}}$, which is obtained by solving Eq. (21) with $c \approx c_{0}$.

\section{The $\gamma_{M}$ and $\gamma_{J}$ wind inputs}

\section{1 $\gamma_{M}$ wind input}

The imaginary part of $c$ gives directly the growth rate of $\eta(x, t)$ :

$\gamma_{\mathrm{M}}=k \Im(c)$.

All the physical quantities derived from the growth rate $\gamma_{\mathrm{M}}$ can be expressed with three parameters: $\delta, \theta_{\mathrm{dw}}$ and $\theta_{\mathrm{fd}}$ (Young, 1997a, b):

$$
\begin{gathered}
\delta=\frac{g h}{U_{1}^{2}}, \quad \theta_{\mathrm{dw}}=\frac{1}{U_{1}} \sqrt{\frac{g}{k}}, \\
\theta_{\mathrm{fd}}=\theta_{\mathrm{dw}} \tanh ^{1 / 2}\left(\frac{\delta}{\theta_{\mathrm{dw}}^{2}}\right) .
\end{gathered}
$$

The dimensionless parameter $\delta^{1 / 2}$, for constant $h$, measures the relative value of the shallow-water speed with respect to $U_{1}$. The parameter $\theta_{\mathrm{dw}}$, equivalent of the wave age in deep water, measures the phase speed relatively to the characteristic wind parameter $U_{1}$ in deep water, and $\theta_{\mathrm{fd}}$ (a finite depth wave age) measures the influence of the finite depth on $\theta_{\mathrm{dw}}$.

In experiments, wether in wave tank or in field, the parameter $C_{\mathrm{p}}$ is used. $C_{\mathrm{p}}$ is the observed phase speed at the peak frequency $\Omega_{\mathrm{p}}$. In this paper we used in Eq. (25) the phase velocity $c=\omega / k$ of one mode instead of $C_{\mathrm{p}}$ or $\Omega_{\mathrm{p}}$. Next we choose dimensionless variables (topped by hats)

$U=U_{1} \hat{U}, \quad \mathcal{W}_{\mathrm{a}}=W_{0} \hat{\mathcal{W}}_{\mathrm{a}}, \quad z=\frac{\hat{z}}{k}$,

$c=U_{1} \hat{c}, \quad t=\frac{U_{1}}{g} \hat{t}$.

Using Eqs. (25) and (26) in Eq. (23) and discarding terms of order two in $s$ we obtain $c$,

$\hat{c}\left(\delta, \theta_{\mathrm{dw}}\right)=\theta_{\mathrm{dw}} T^{1 / 2}\left(1-\frac{s}{2}\right)+\frac{s}{2}\left\{T \hat{I}_{1}-\theta_{\mathrm{dw}} T^{3 / 2} \hat{I}_{2}\right\}$,

where $T=\tanh \frac{\delta}{\theta_{\mathrm{dw}}^{2}}$. With $e^{\gamma \mathrm{M} t}$, we obtain the dimensionless growth rate $\hat{\gamma}_{\mathrm{M}}=\frac{U_{1}}{g} \gamma_{\mathrm{M}}$ as

$\hat{\gamma}_{\mathrm{M}}=\frac{s}{2}\left\{\frac{T \Im\left(I_{1}\right)}{\theta_{\mathrm{dw}}^{2}}-\frac{T^{3 / 2} \Im\left(I_{2}\right)}{\theta_{\mathrm{dw}}}\right\}$.
We have the following transformation rule between $\beta$ and the dimensionless $\hat{\gamma}_{M}$ (Montalvo et al., 2013):

$\beta=\frac{2 \hat{\gamma_{M}}}{s} \theta_{\mathrm{dw}}^{3} T^{1 / 2}$.

The dimensional mean energy growth rate,

$\Gamma_{\mathrm{M}}=\frac{1}{\langle E\rangle}\left\langle\frac{\partial E}{\partial t}\right\rangle$,

can then be written as a function of $\beta$ :

$\Gamma_{\mathrm{M}}=s \beta U_{1}^{2}\left(\frac{k^{3}}{g}\right)^{1 / 2} \operatorname{coth}^{1 / 2}(k h)$.

\section{$3.2 \gamma_{\mathrm{J}}$ wind input}

Jeffreys (1926) established that the pressure component acting on a surface wave can be written as

$P_{\mathrm{a}, \text { Jeffreys }}=P_{\mathrm{a}, \mathrm{J}}=S \rho_{\mathrm{a}}\left(U_{10}-c\right)^{2} \eta_{x}$,

where $\eta$ is the free surface elevation; $S$ is the sheltering coefficient, always lower than unity; and $U_{10}$ is the $10 \mathrm{~m}$ wind velocity. This is only valid when the wave slope is larger than a certain critical value. Such a pressure gradient can also be obtained when the boundary layer thickness varies from one side of the wave crest to another, thickening on the leeward slope and resulting in a non-separated sheltering (Belcher and Hunt, 1993). We do not consider variations of the boundary layer here. Then Jeffreys assumed that the rate of work transfer from the wind to the wave is (average with respect to time)

$\left\langle\frac{\partial E}{\partial t}\right\rangle=\left\langle P_{\mathrm{a}, \mathrm{J}} \cdot\left(-\frac{\partial \eta}{\partial t}\right)\right\rangle$.

Our study being perturbative, we do not have any information on the possible value of $\eta$ whatsoever. We assume a sinusoidal wave of the form $\eta=\eta_{0} \cos k(x-c t), \eta_{0}$ being the wave amplitude. With this, and recalling that the mean wave energy is $\langle E\rangle=\rho_{\mathrm{w}} g \frac{\eta_{0}^{2}}{2}$, we can deduce the energy growth rate expression

$\Gamma_{\mathrm{J}}=\frac{k^{2}}{g} \operatorname{cs} S\left(U_{10}-c\right)^{2}$.

Now, in order to transform $U_{10}$ into $U_{1}$, the relevant wind scale, we use the wind-stress coefficient $C_{10}$, as defined by Wu (1982) and the Charnock relation (Charnock, 1955):

$$
\begin{aligned}
& C_{10}=\left(0.8+0.065 U_{10}\right) \times 10^{-3}=\frac{u_{*}^{2}}{U_{10}^{2}}, \\
& U_{1}=\frac{U_{10}}{\kappa} \sqrt{C_{10}} .
\end{aligned}
$$

$\mathrm{Wu}$ (1982) showed that the empirical Eqs. (35) and (36) proposed for light winds are even applicable in hurricane 


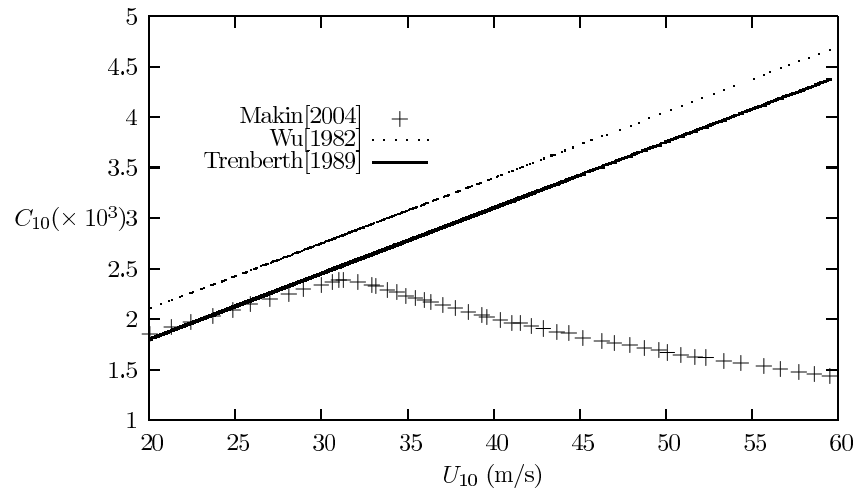

Fig. 1. The drag coefficient versus the $U_{10}$ wind velocity. As we can see, it follows a linear progression until its maximum around $30 \mathrm{~m} \mathrm{~s}^{-1}$, then decreases. So, a linear parametrization of $C_{10}$ is a reasonable approximation.

conditions. However, above a $30 \mathrm{~m} \mathrm{~s}^{-1}$ limit, the drag coefficient drops off the $U_{10}$ linear progression in Eq. (35), as we can see in Fig. 1. This phenomenon reported in Powell et al. (2003) is due to the droplet saturation in a suspension layer above the sea surface. Even though it is possible to use the model developed in Makin (2004) to calculate the correct drag coefficient and friction velocity, flow separation is likely to occur at wind speeds that high, preventing Miles' mechanism from acting. Hence, we keep the range of wind speeds below this limit for computation and we can use Eqs. (35) and (36).

\section{Results}

In the following Sects. 4.1, 4.2, 4.3 and 5 we present (i) the evolution of the finite depth wind-wave input $\beta$ with $\theta_{\mathrm{dw}}$ for $k h$ constant; (ii) its evolution, as a function of wind velocity and wave age, with $h$ constant; (iii) a comparison between the Miles and Jeffreys mechanism for finite depth; and (iv) a wind-forced NLS equation in finite depth.

After recalling several approximations, we are going to work with the finite depth $\beta$-Miles wind input instead of $\hat{\gamma}_{\mathrm{M}}$.

\subsection{The finite depth $\beta$-Miles with constant $k h$}

First, we plot in Fig. 2 the evolution of the growth rate with $\theta_{\mathrm{fd}}$ for several constant values of the $k h$ parameter. Neither $k$ nor $h$ are constants,

- for large values of the theoretical wave age $\theta_{\mathrm{fd}}$, the values of $\beta$ are in the deep-water limit,

- from small to intermediate values of $\theta_{\mathrm{fd}}$ the values of $\beta$ are lower than in the deep-water limit and

$$
\frac{\mathrm{d} \beta}{\mathrm{d} \theta_{\mathrm{fd}}} \sim 0 \text {. }
$$

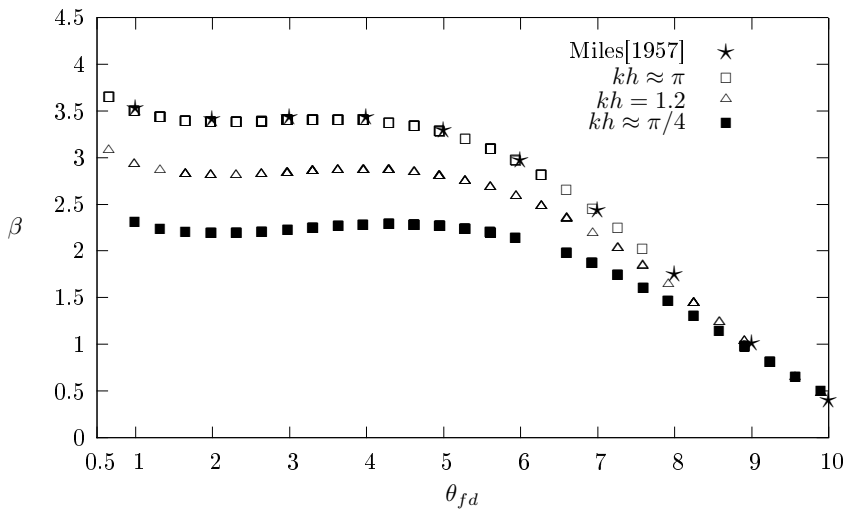

Fig. 2. Miles' $\beta$ vs $\theta_{\mathrm{fd}}$. For the deep-water limit $k h \approx \pi$ our results fit the Miles curve. $k h<\pi / 4$ corresponds to shallow water that is beyond the range of validity of our model. An intermediate value of $k h$ is included, and we see that $\beta$ is less than in the deep-water limit.

The small values of $\theta_{\mathrm{fd}}$ seem constrained. In fact, because all the curves are calculated with the same parameter space $h \in[0.1 \mathrm{~m}, 18 \mathrm{~m}]$, different bounds on $k h$ give different bounds on $k$, and subsequently on $\theta_{\mathrm{fd}}$. For the deepwater limit $k h \approx \pi$ we rediscover Miles' result, and below the shallow-water limit $k h \approx \pi / 4$ we are beyond the validity of the model.

\subsection{The finite depth $\beta$-Miles from weak to moderate winds with $h$ constant}

In deep water, we have the classical Miles curve $\beta\left(\theta_{\mathrm{dw}}\right)$. Herein, the introduction of the parameter $\delta$ transforms the unique curve of wave growth rate in families of curves $\beta\left(\theta_{\mathrm{fd}}\right)$ indexed by $\delta=g h / U_{1}^{2}$, i.e. a curve for each value of $\delta$. Two types of families are possible:

- a family of $\beta$ curves against $\theta_{\mathrm{fd}}$ indexed by $h$ with $U_{1}$ constant;

- a family of $\beta$ curves against $\theta_{\mathrm{fd}}$ indexed by $U_{1}$, with $h$ constant.

The first one was studied in Montalvo et al. (2013). In this work, and for the first time, we presented curves of wave growth evolution as a function of $U_{1}$ with constant depth $h$. Figures 3, 4 and 5 show $\beta$ curves for constant $h$ as a function of $\theta_{\mathrm{fd}}$, for friction velocities $U_{1}$ from 0.5 to $2.5 \mathrm{~m} \mathrm{~s}^{-1}$. More specifically, this denomination refers to a $10 \mathrm{~m}$ wind velocity, namely $U_{10}$, such that

$5<U_{10}<22 \mathrm{~m} \mathrm{~s}^{-1}$.

One can switch from $U_{1}$ to $U_{10}$ using simply Eq. (36). From now on, we will refer to $U_{10}$ only. The curves show that 


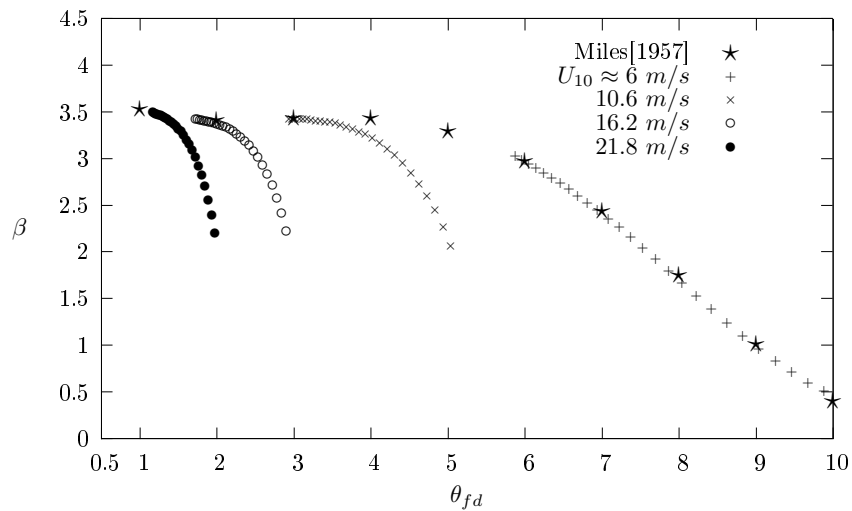

Fig. 3. $\beta$ vs. $\theta_{\mathrm{fd}}$, the wave-age-like parameter. The water depth is $h=3 \mathrm{~m}$ for all curves above. For this depth, all $10 \mathrm{~m}$ wind speeds account for early drops in the growth rate. The deep-water limit $k h \rightarrow \infty$, originally computed by Miles, is plotted for comparison. For the lower wind speed, the growth drop occurs closer to deep water. Although $U_{10}=6 \mathrm{~m} \mathrm{~s}^{-1}$ gives a deep-water-like behaviour, we see that stronger winds imply early (wavelength-wise) drops in the growth rate.

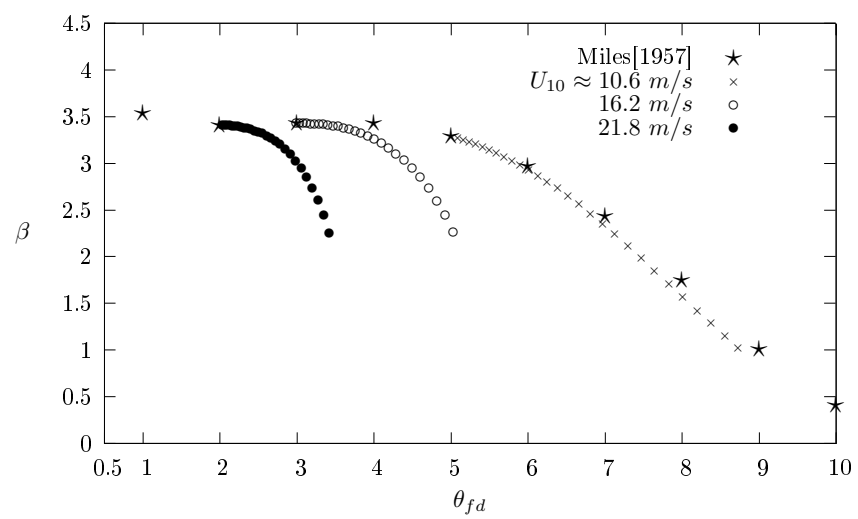

Fig. 4. Same as Fig. 3 with $h=9 \mathrm{~m}$. The lowest wind speed $U_{10} \approx$ $6.0 \mathrm{~m} \mathrm{~s}^{-1}$ is not shown, as the next one already gives us a deepwater limit.

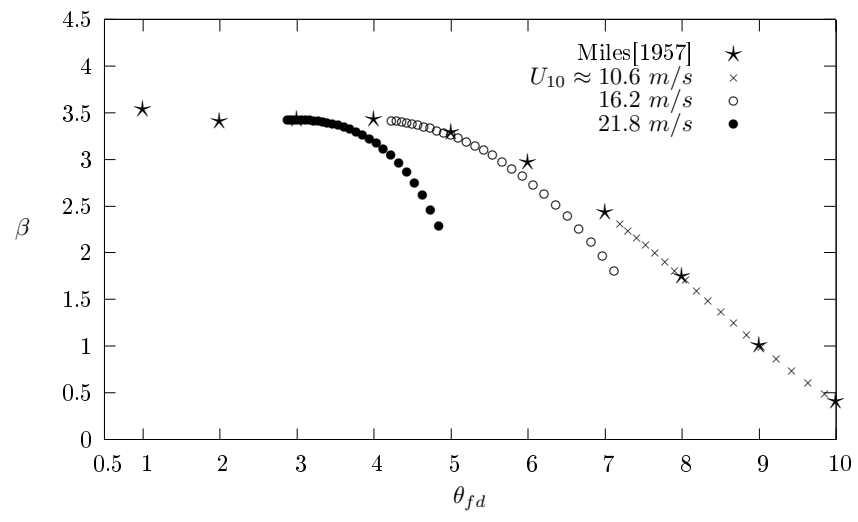

Fig. 5. Same as Fig. 4 with $h=18 \mathrm{~m}$.
- no matter what the values of wind velocities are, at small enough wave age $\theta_{\mathrm{fd}}$ the growth rate $\beta$ satisfies the known deep-water limit;

- the consequences of finite $h$ are visible as $\theta_{\mathrm{fd}}$ augments. The coefficient $\beta$ is lesser than in the deepwater limit. Furthermore, if the finite depth wave age $\theta_{\mathrm{fd}}$ is kept constant, the growth rate $\beta$ decreases as the wind speed $U_{10}$ augments.

Each $U_{10}$ curve approaches its own theoretical $\theta_{\mathrm{fd}}$-limited growth as $\beta$ goes to zero (no energy transfer). Then, the wave propagates steadily without changing its amplitude. The $\theta_{\mathrm{fd}}$ at which this happens is lower as the wind speed augments. Consequently, developed seas are reached faster under moderate winds than under weak winds. The evolution of $\beta$ under wind intensity and wave age shown in Figs. 3, 4, and 5 is not a dynamical one, but rather a collection of wave snapshots taken at every step of the growth in height and age.

\subsection{Comparison $\gamma_{M}$ versus $\gamma_{J}$}

Very recently, Tian and Choi (2013) investigated experimentally and numerically the evolution of deep-water waves interacting with wind, with breaking effects. They discussed the relative importance of Miles' and Jeffreys' models and showed that Miles' model may be used for waves of moderate wave steepness under weak to moderate wind forcing, whereas for steep waves under strong wind forcing both mechanisms may have to be considered. In this section we desire to measure the relative importance of Miles' mechanism versus Jeffreys' mechanism in finite depth. To do that, we follow the idea in Touboul and Kharif (2006). Taking the derived growth rates from Sects. 3.1 and 3.2, one can establish the ratio between them. It reads, with only nondimensional parameters,

$R=\frac{\Gamma_{\mathrm{J}}}{\Gamma_{\mathrm{M}}}=\frac{t_{\mathrm{M}}}{t_{\mathrm{J}}}=\frac{S T}{\beta}\left(\frac{\kappa}{\sqrt{C_{10}}}-\theta_{\mathrm{fd}}\right)$,

where $t_{\mathrm{M}}=\Gamma_{\mathrm{M}}^{-1}$ and $t_{\mathrm{J}}=\Gamma_{\mathrm{J}}^{-1}$ are the characteristic timescales of growth for the Miles and Jeffreys mechanism. Hence, we can calculate $R\left(U_{10}, \theta_{\mathrm{fd}}\right)$ to study the evolution of this ratio with the theoretical wave age, for different values of the wind speed. Each point in the $\left(\theta_{\mathrm{fd}}, U_{10}\right)$ plane corresponds to a water depth $h$ between $3 \mathrm{~m}$ and $18 \mathrm{~m}$ and a dispersive parameter $k h \in\left[\frac{\pi}{4} ; \pi\right]$. These boundaries on $k h$ correspond to the shallow-water and deep-water limits, as discussed previously. The results are shown in Fig. 6. When this ratio is significantly greater than unity, it means that the Jeffreys mechanism acts faster, and dominates. Conversely, Miles' mechanism is dominant for values lower than unity. One can observe in finite depth that the Miles mechanism is dominant for mature waves whereas the Jeffreys mechanism is dominant for younger waves. This comparison stands as long as the wave is steep enough to induce a sheltering, as discussed before. 


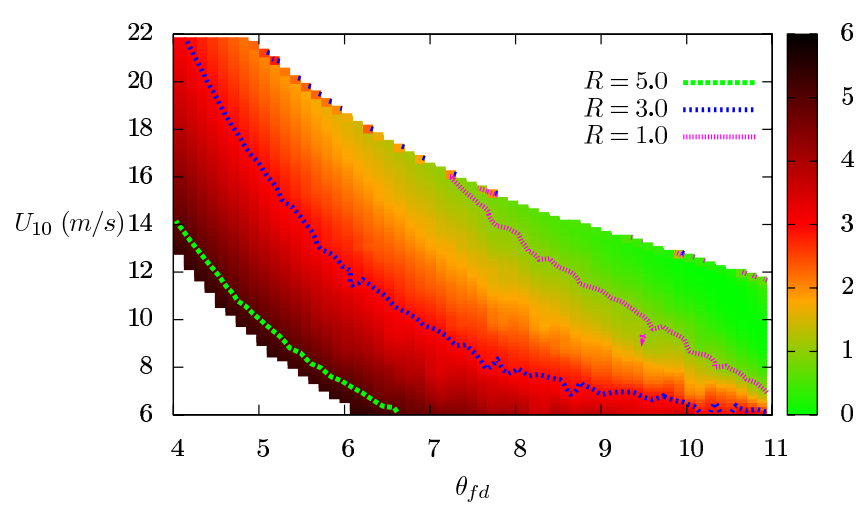

Fig. 6. $\left(U_{10}, \theta_{\mathrm{fd}}\right)$ parameter space for continuously varying values of $R=\Gamma_{\mathrm{J}} / \Gamma_{\mathrm{M}}$. In the domain defined by $R<1$, the Miles mechanism is dominant, whereas for $R>1$ the Jeffreys mechanism is dominant.

\section{Wind-forced nonlinear Schrödinger equation in finite depth}

Let us consider the air/water system from a quasi-linear point of view; i.e. the water dynamics is considered nonlinear and irrotational and, as in Miles' theory, the airflow is kept linear. So with this assumption the complete irrotational Euler equations and boundary conditions in terms of the velocity potential $\phi(x, z, t)$ are

$\phi_{\mathrm{xx}}+\phi_{\mathrm{zz}}=0$ for $-h \leq z \leq \eta(x, t)$,

$\phi_{z}=0$ for $z=-h$,

$\eta_{t}+\phi_{x} \eta_{x}-\phi_{z}=0$ for $z=\eta(x, t)$,

$\phi_{t}+\frac{1}{2} \phi_{x}^{2}+\frac{1}{2} \phi_{z}^{2}+g \eta=-\frac{1}{\rho_{\mathrm{w}}} P_{\mathrm{a}}$ for $z=\eta(x, t)$.

In Miless' theory of wave generation (Miles, 1957, 1997), the complex air pressure $P_{\mathrm{a}}$ can be separated into two components, one in phase and one in quadrature with the free surface $\eta$. A phase shift between those two quantities is necessary to transfer energy from the airflow to the wave field. The transfer is only due to the part of $P_{\mathrm{a}}$ in quadrature with $\eta$. Hence, we will deal only with the acting pressure component, that is,

$P_{\mathrm{a}}(x, t)=\rho_{\mathrm{a}} \beta U_{1}^{2} \eta_{x}(x, t)$,

so that the modified Bernoulli equation reads

$\phi_{t}+\frac{1}{2} \phi_{x}^{2}+\frac{1}{2} \phi_{z}^{2}+g \eta=-s \beta U_{1}^{2} \eta_{x} \quad$ for $\quad z=\eta(x, t)$.

From Eqs. (38), (39), (40), and (43) we find a wind-forced finite depth NLS equation for $\eta$ as a function of the standard slow space and time variables $\xi=\varepsilon\left(x-c_{\mathrm{g}} t\right)$ and $v=\varepsilon^{2} t$, with $\varepsilon \ll 1$ and $c_{\mathrm{g}}$ the group velocity. The perturbed NLS equation reads

$i \eta_{v}+a \eta_{\xi \xi}+b|\eta|^{2} \eta=i d \eta$ with $c_{\mathrm{g}}, a, b$ and $d$ given by Eqs. (45), (46), (47), and (48):

$$
\begin{aligned}
c_{\mathrm{g}}= & \frac{c}{2}[1+2 k h / \sinh (2 k h)], \\
a= & -\frac{c_{\mathrm{g}}^{2}-g h\left[1-k h T\left(1-T^{2}\right)\right]}{2 \omega}, \\
b= & \frac{k^{4} c^{2}}{4 \omega T^{2}}\left[\frac{9}{T^{2}}-12+13 T^{2}\right. \\
& \left.-2 T^{4}-\frac{2\left[2 c+c_{\mathrm{g}}\left(1-T^{2}\right)\right]^{2}}{g h-c_{\mathrm{g}}^{2}}\right], \\
d= & s \frac{\beta}{2} \frac{U_{1}^{2}}{c^{2}} T \omega .
\end{aligned}
$$

For more information about the derivation of the coefficients $a$ and $b$ see Thomas et al. (2012). To derive a dimensionless wind-forced NLS equation we use Eq. (26) and we obtain in the original laboratory variables $x$ and $t$ (after a Galilean transformation in order to eliminate the linear term $c_{\mathrm{g}} \eta_{x}$ and dropping the hats)

$i \eta_{t}+A \eta_{\mathrm{xx}}+B|\eta|^{2} \eta=i D \eta$,

with $c_{\mathrm{g}}, A, B$, and $D$ now given by Eqs. (50), (51), (52) and (53):

$$
\begin{aligned}
c_{\mathrm{g}}= & \frac{1}{2 \theta_{\mathrm{fd}}}\left[1+\frac{\delta}{\theta_{\mathrm{dw}}^{2}} \frac{1-T^{2}}{T}\right], \\
A= & -\frac{c_{\mathrm{g}}^{2}-\delta\left[1-\delta \theta_{\mathrm{dw}}^{-2}\left(1-T^{2}\right)\right]}{2 \theta_{\mathrm{fd}} \theta_{\mathrm{dw}}^{2}}, \\
B= & \frac{1}{4 T^{2} \theta_{\mathrm{fd}}^{3} \theta_{\mathrm{dw}}^{2}}\left[\frac{9}{T^{2}}-12+13 T^{2}\right. \\
& \left.-2 T^{4}-\frac{2\left[2 \theta_{\mathrm{fd}}^{-1}+c_{\mathrm{g}}\left(1-T^{2}\right)\right]^{2}}{\delta-c_{\mathrm{g}}^{2}}\right],
\end{aligned}
$$

$D=s \frac{\beta}{2} \frac{T^{1 / 2}}{\theta_{\mathrm{dw}}^{3}}$.

Equation (49) is a wind-forced finite depth NLS equation in dimensionless variables.

\subsection{The Akhmediev, Peregrine and Ma solutions for weak wind inputs in finite depth}

The classical nonlinear Schrödinger equation provides a model for freak waves; see for example Touboul and Kharif (2006), Touboul et al. (2008), Kharif et al. (2008) and references therein. The wind-forced nonlinear Schrödinger equation allows the study of the wind influence on the freak wave dynamics (Touboul and Kharif, 2006; Touboul et al., 2008; Kharif et al., 2008; Onorato and Proment, 2012). Previous authors have carried out such studies in deep water. 
Didenkulova, Nikolkina and Pelinovsky (2013) studied the Peregrine breather in water of finite depth without the wind influence. The present work allows, for the first time, similar studies in finite depth with the right Miles growth rates.

In the following we are going only to consider the socalled focusing NLS equation, i.e. positives $A$ and $B$. Introducing $\eta^{\prime}$ and $x^{\prime}$ as

$\eta^{\prime}=\sqrt{B} \eta, \quad x^{\prime}=\frac{x}{\sqrt{A}}$,

Eq (49) transforms, dropping the primes, into

$i \eta_{t}+\eta_{\mathrm{xx}}+|\eta|^{2} \eta=i D \eta$.

Introducing a function $M(x, t)$ as

$M(x, t)=\eta(x, t) \exp (-D t)$,

we obtain from Eq. (54)

$i M_{t}+M_{\mathrm{xx}}+\exp (2 D t)|M|^{2} M=0$.

In order to reduce Eq. (54) into the standard form of the NLS with constant coefficients we proceed in the following way. First of all we consider the wind forcing $2 D t$ to be weak, such that the exponential can be approximated so we have

$i M_{t}+M_{\mathrm{xx}}+n|M|^{2} M=0, \quad n=n(t)=\frac{1}{1-2 D t}$.

Now with a change of coordinates from $(x, t)$ to $(z, \tau)$ defined by

$z(x, t)=x n(t), \quad \tau(t)=\operatorname{tn}(t)$,

and scaling the wave envelope as (Onorato and Proment, 2012)

$M(z, \tau)=\Psi(z, \tau) \sqrt{n(\tau)} \exp \left(\frac{-i D z^{2}}{n(\tau)}\right)$,

we reduce Eq. (57) to the standard focusing equation for $\Psi(z, \tau)$ :

$i \Psi_{\tau}+\Psi_{\mathrm{zz}}+|\Psi|^{2} \Psi=0$.

Equation (60) admits well-known breather solutions that are simple analytical prototypes for rogue wave events. They are the Akhmediev $\left(\Psi_{\mathrm{A}}\right)$ (Akhmediev et al., 1987), the Peregrine $\left(\Psi_{\mathrm{P}}\right)$ (Peregrine, 1983) and the Kuznetsov-Ma $\left(\Psi_{\mathrm{M}}\right)$ (Ma, 1979) breather solutions.

Dysthe and Trulsen (1999) investigated whether freak waves in deep water could be modelled by $\Psi_{\mathrm{A}}, \Psi_{\mathrm{P}}$ or by $\Psi_{\mathrm{M}}$. Onorato and Proment (2012) considered the influence of weak wind forcing and dissipation on these $\Psi_{\mathrm{A}}, \Psi_{\mathrm{P}}$ or $\Psi_{\mathrm{M}}$ solutions in deep water.
The present work allows us to go ahead and to exhibit expressions for $\Psi_{\mathrm{A}}, \Psi_{\mathrm{P}}$ and $\Psi_{\mathrm{M}}$ under the influence of weak wind forcing in finite depth $h$ given by the extended Miles mechanism. These solutions read (Dysthe and Trulsen, 1999)

$\eta_{A}=P(\tau)\left\{\frac{\cosh (\Omega \tau-2 i \omega)-\cos (\omega) \cos (p z)}{\cosh (\Omega \tau)-\cos (\omega) \cos (p z)}\right\}$,

with $p=2 \sin (\omega), \Omega=2 \sin (2 \omega), \omega$ real and $p$ related to the spatial period $2 \pi / p$,

$\eta_{P}=P(\tau)\left\{1-\frac{4(1+4 i \tau)}{1+4 z^{2}+16 \tau^{2}}\right\}$,

$\eta_{\mathrm{M}}=P(\tau)\left\{\frac{\cos (\Omega \tau-2 i \omega)-\cosh (\omega) \cosh (p z)}{\cosh (\Omega \tau)-\cos (\omega) \cos (p z)}\right\}$,

with $p=2 \sinh (\omega), \Omega=2 \sinh (2 \omega)$ and $\Omega$ real and related to the time period $2 \pi / \Omega$ and

$P(\tau)=n(\tau) \exp \left[\frac{-i D z^{2}}{n(\tau)}\right] \exp [2 i \tau]$.

A more detailed analytical and numerical analysis in terms of $x$ and $t$ of Eq. (49) will be developed in a future work.

\section{Conclusions}

We have extended the well-known Miles theory to the finite depth case under breeze to moderate wind conditions. We have linearized the equations of motion governing the dynamics of the air/water interface problem in finite depth, and we have investigated the linear instability in time of a normal Fourier mode of wave number $k$ in Miles' and Jeffreys' mechanisms in finite depth. For the Miles mechanism we have shown that normal modes are unstable and grow exponentially in time as

$\exp \left[\frac{s \beta}{2 \theta_{\mathrm{fd}}^{3} T^{1 / 2}}\right] t$,

with $\beta$ the finite depth Miles coefficient. The curves of $\beta$ against $\theta_{\mathrm{fd}}$ with $k h$ constant showed essentially that the values of $\beta$ remain smaller than those corresponding to the deep-water limit $\forall \theta_{\mathrm{fd}}$. Wind effects on the temporal growth have been discussed. From a comparison between the growth rates $\gamma_{\mathrm{M}}$ and $\gamma_{\mathrm{J}}$ a diagram in the $\left(\theta_{\mathrm{fd}}, U_{10}\right)$ plane displays the domains where the Miles mechanism $(R<1)$ or the Jeffreys mechanism $(R>1)$ is dominant.

We have derived for the first time a wind-forced finite depth nonlinear Schrödinger equation. The wind forcing is based on the Miles theory extended to finite depth. This equation admits the Akhmediev, Peregrine and Kuznetsov-Ma breather solutions for weak wind input in finite depth. 
In this paper we have used the conventional finite depth NLS second-order envelope equation under the wind action. The third-order finite depth NLS equations introduced by Slunyaev (2005) could improve the results.

Other factors influence the mechanisms of wave growth under wind action, in finite depth: for instance, time variations of wind speed and wind direction, the bathymetry effects in the field, loss of energy by bottom friction, airflowinduced surface drifts, turbulence, nonlinear interactions between waves, flow separation, dissipation due to white capping and so on.

The scope of this paper is not to address all of these phenomena, and they will be treated in a future work. Nevertheless, we believe that this work could be useful for the understanding of wave generation in finite-depth situations, namely in the coastal zone. The present theory is the first step towards more accurate freak wave models in finite depth.

Acknowledgements. P. Montalvo thanks Labex NUMEV (Digital and Hardware Solutions, Modeling for the Environment and Life Sciences) for partial financial support.

M. A. Manna thanks the PVE program (Pesquisador Visitante Especial, CAPES/BRASIL).

Edited by: E. Pelinovsky

Reviewed by: two anonymous referees

\section{References}

Akhmediev, N. N., Eleonskii, V. M., and Kulagin, N. E.: Exact first-order solution on the nonlinear Schrödinger equation, Theor. Math. Phys., 72, 809-818, 1987.

Banner, M. and Melville, W.: On the separation of air flow over water waves, J. Fluid Mech., 77, 825-842, 1976.

Belcher, S. E. and Hunt, J. C. R.: Turbulent shear flow over slowly moving waves, J. Fluid Mech., 251, 109-148., 1993.

Charnock, H.: Wind stress on a water surface, Q. J. Roy. Meteor. Soc., 81, 639-640, 1955.

Didenkulova, I. I., Nikolkina, I. F., and Pelinovsky, E. N.: Rogue Waves in the Basin of Intermediate Depth and the Possibility of Their Formation Due to the Modulational Instability, JETP Letters, 97, 194-198, 2013.

Dysthe, K. B. and Trulsen, K.: Note on Breather Type Solutions of the NLS as Models for Freak-Waves, Phys. Scripta, T82, 48-52, 1999.

Garratt, J., Hess, G., Physick, W., and Bougeault, P.: The atmospheric boundary layer advances in knowledge and application, Bound.-Lay. Meteorol., 78, 9-37, 1996.

Janssen, P.: The Interaction of Ocean Waves and Wind, Cambridge University Press, UK, 2004.

Jeffreys, H.: On the formation of water waves by wind, P. R. Soc. Lond. A-Conta., 107, 189-206, 1925.

Jeffreys, H.: On the formation of water waves by wind (Second paper), P. R. Soc. Lond. A-Conta., 110, 241-247, 1926.
Kawai, S.: Structure of air flow separation over wind wave crests, Bound.-Lay. Meteorol., 23, 503-521, 1982.

Kharif, C., Giovanangeli, J.-P., Touboul., C., Grade, L., and Pelinovsky, E.: Influence of wind on extreme wave events: experimental and numerical approaches, J. Fluid Mech., 594, 209-247, 2008.

Lighthill, J.: Waves in Fluids, Cambridge University Press, UK, 1978.

Ma, Y.: The perturbed plane-wave solutions of the cubic Schrödinger equation, Stud. Appl. Math, 60, 43-58, 1979.

Makin, V.: A note on the drag of the sea surface at hurricane winds, Bound.-Lay. Meteorol., 115, 169-176, 2004.

Miles, J.: On the generation of surface waves by shear flows, J. Fluid Mech., 3, 185-204, 1957.

Miles, J.: Generation of surface waves by winds, Appl. Mech. Rev, 50-7, R5-R9, 1997.

Montalvo, P., Dorignac, J., Manna, M., Kharif, C., and Branger, H.: Growth of surface wind-waves in water of finite depth, a theoretical approach, Coast. Eng., 77, 49-56, doi:10.1016/j.coastaleng.2013.02.008, 2013.

Onorato, M. and Proment, D.: Approximate rogue wave solutions of the forced and damped Nonlinear Schrödinger Equation for water waves, Phys. Lett. A, 376, 3057-3059, 2012.

Peregrine, D.: Water waves, nonlinear Schrödinger equation and their solutions, J. Austral. Math. Soc. Ser. B, 25, 16-43, 1983.

Phillips, O.: On the generation of waves by turbulent wind, J. Fluid Mech., 2, 417-445, 1957.

Powell, M., Vickery, P., and Reinhold, T.: Reduced drag coefficient for high speeds in tropical cyclones, Nature, 422, 279-283, 2003.

Rayleigh, L.: On the stability or instability of certain fluid motions, P. Lond. Math. Soc., 11, 57-70, 1880.

Reul, N., Branger, H., and Giovanangeli, J.-P.: Air flow structure over short-gravity breaking water waves, Bound.-Lay. Meteorol., 126, 477-505, 2008.

Slunyaev, A. V.: A High-Order Nonlinear Envelope Equation For Gravity Waves in Finite-Depth Water, J. Exp. Theor; Phys., 101, 926-941, 2005.

Thomas, R., Kharif, C., and Manna, M. A.: A nonlinear Schrödinger equation for waves on finite depth with constant vorticity, Phys. Fluids, 24, 138-149, doi:10.1063/1.4768530, 2012.

Tian, Z. and Choi, W.: Evolution of deep-water waves under wind forcing and wave breaking effects: numerical simulations and experimental assessment, Eur. J. Mech. B-Fluid., 41, 11-22, doi:10.1016/j.euromechflu.2013.04.001, 2013.

Touboul, J. and Kharif, C.: On the interaction of wind and extreme gravity waves due to modulational instability, Phys. Fluids, 18, 108103-1-108103-4, doi:10.063/1.2374845, 2006.

Touboul, J., Kharif, C., Pelinovsky, E., and Giovanangeli, J.-P.: On the interaction of wind and steep gravity wave groups using Miles' and Jeffreys' mechanisms, Nonlin. Processes Geophys., 15, 1023-1031, doi:10.5194/npg-15-1023-2008, 2008.

$\mathrm{Wu}, \mathrm{J} .:$ Wind-stress coefficients over sea surface from breeze to hurricane, J. Geophys. Res., 87, 9704-9706, 1982.

Young, I.: The growth rate of finite depth wind-generated waves, Coastal Eng., 32, 181-195, 1997a.

Young, I.: Wind Generated Ocean WWaves, Elsevier, Kidlington, Oxford, UK, $1997 \mathrm{~b}$. 